\title{
AUTOMATIZACIÓN DEL PROCESO DE GENERACIÓN DEL SEGURO ESTUDIANTIL, APLICANDO LAS TECNOLOGÍAS RFID Y GPRS
}

(Automation of the process of generation of the students insurance, applying RFID and GPRS technologies)

\section{Nelson Barrera Lombana, Luis Ariel Mesa Mesa, Jorge Julián Moreno Rubio}

Grupo de Investigación en Robótica y Automatización Industrial GIRA, Universidad Pedagógica y Tecnológica de Colombia UPTC. Correos: barreranelson@hotmail.com, ariel.mesa@uptc.edu.co,

jorgejulian.moreno@uptc.edu.co

(Recibido19 de Noviembre de 2012 y aceptado 13 de Marzo de 2013)

\begin{abstract}
Resumen:
En este artículo se presenta la descripción del diseño e implementación de un sistema que permite el despliegue de un servicio de consultas de diversos parámetros a un servidor de Internet, utilizando un módem GSM, sistemas de intercambio de información a través de Internet (ISS) e identificación de usuarios mediante radiofrecuencia (RFID). La aplicación se valida por su uso en la automatización del proceso de generación del seguro estudiantil, y se emplean como plataforma el software y el hardware desarrollados por el Grupo de Investigación en Robótica y Automatización Industrial GIRA de la UPTC.
\end{abstract}

Palabras clave: módem GPRS, RFID, microcontrolador, aplicaciones web.

\section{Abstract:}

This article presents the description of the design and implementation of a system which allows the fulfilment of a consultation service on various parameters to a web server using a GSM modem, exchanging information systems over the Internet (ISS) and radio-frequency identification (RFID). The application validates for its use in automation of the process of generation of the student insurance, and hardware and software, developed by the Research Group in Robotics and Industrial Automation GIRA of UPTC, are used as a platform.

Keywords: modem, GPRS, RFID, microcontroller, web applications.

\section{INTRODUCCIÓN}

En el presente artículo se describe un dispositivo electrónico que ha sido diseñado y construido con el objetivo de automatizar el proceso de generación del seguro estudiantil, que en la actualidad es diligenciado de forma manual. De esta forma, se busca suplir la necesidad de agilizar los procesos de matrícula de los estudiantes y disminuir las congestiones generadas por la forma anticuada en que se lleva a cabo dicho proceso en la actualidad.

Para dar solución a este problema, se sugiere el uso de las tecnologías de identificación de los estudiantes por radiofrecuencia (Radio Frecuency Identification RFID); método que emplea dispositivos con enlaces inalámbricos, los cuales permiten el almacenamiento (en dispositivos receptores transmisores: transceiver) y la recuperación de datos, son de bajo costo y se denominan etiquetas o tag. Estos tag son sistemas electrónicos de reducidas dimensiones, que pueden ser encapsulados en circuitos integrados, adhesivos plásticos, llaveros y carnés, entre otros. Así, dadas sus múltiples presentaciones, estos pueden ser adheridos a diversos productos o acompañar a una persona, permitiendo que el portador del tag sea identificado por un receptor RFID, a través del enlace inalámbrico que tiene asociada cada etiqueta (tranceiver).
Los datos colectados por el circuito identificador RFID, son enviados a través del servicio general de paquetes (General Packet Radio Service GPRS), lo que da la posibilidad de consultar esta información vía Internet, en cualquier momento y de forma simple, siempre que se cuente con una dirección IP (Internet Protocol), haciendo uso de la posibilidad que dan los operadores de las redes de comunicación móvil, de generar costos por volumen de información enviada, dejando de lado el cobro por tiempo de conexión. De esta forma, se logra agilidad en el manejo de los datos y bajo costo en el transporte de estos (Floyd, 2005).

La tecnología RFID y los tag permiten que el estudiante se identifique sin necesidad de un carné convencional y sin personal que atienda sus peticiones. De esta forma, la etiqueta identifica al educando, la información es llevada a una base de datos mediante la tecnología GPRS, y un servidor web procesa la información y responde las peticiones que realicen los educandos. Con este sistema es posible automatizar múltiples procesos, como la compra del almuerzo, de fotocopias y un sinnúmero de servicios que ofrece la universidad y cuyo costo debe ser asumido por el estudiante. En este caso, el estudiante puede beneficiarse de las ventajas del sistema, al pagar de forma previa los costos de estos servicios, los cuales serán cargados en el tag RFID correspondiente.
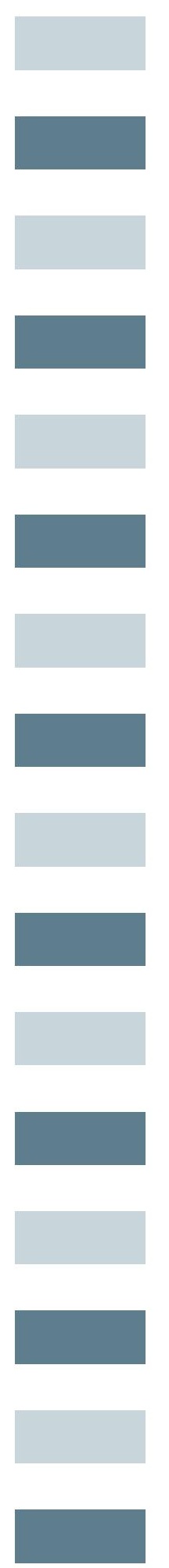
Así, el usuario del sistema puede cargar su tarjeta y disfrutar de diferentes servicios. Entonces, cada vez que haga uso de esta, el sistema lo identificará, hará la consulta del saldo del estudiante en el servidor web mediante GPRS, y si este es suficiente, atenderá la petición y descargará el costo de su cuenta (en definitiva es un sistema de cobro similar al que emplea la telefonía celular prepago). El sistema propuesto aparece en la Figura 1.

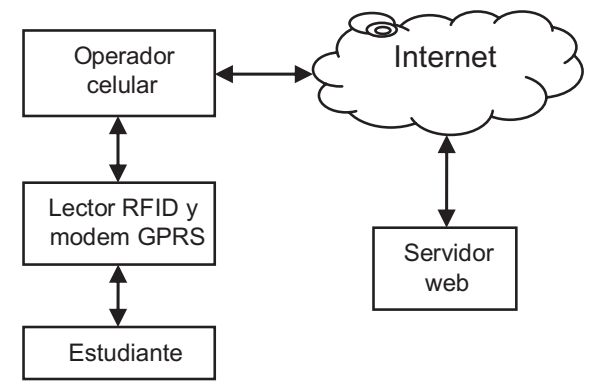

Figura 1. Descripción general del sistema elaborado.

Dado que el sistema es un prototipo, se propone usarlo en el trámite del pago del seguro estudiantil, ya que este genera pocas consultas y no es un proceso crítico que ocasione un elevado tráfico de datos y que pueda llevar a que el sistema colapse. De esta forma, si el sistema pasa las pruebas con este servicio, posteriormente podrá ser aplicado a un mayor número de actividades de forma fiable, eficaz y eficiente.

Para el desarrollo de la propuesta, se plantean las siguientes seis etapas que hacen posible la construcción del sistema propuesto:

Documentación que permita conocer y manipular de forma instrumental y simbólica los sistemas basados en las tecnologías RFID y GPRS, seleccionando los dispositivos más adecuados disponibles en el mercado, desde la perspectiva económica y funcional, de acuerdo con las necesidades y los recursos con que cuenta el grupo de investigación GIRA-UPTC (Garza, 2006).

Análisis para determinar las necesidades del hardware requerido, a fin de lograr que los sistemas RFID y GPRS puedan interactuar de forma adecuada con un servidor web y con aplicaciones para ordenador que posibiliten procesar la información recogida de los tag que portan los estudiantes y atender las peticiones de estos.

- Diseño del hardware y del software sobre el que se materializará la propuesta.

- Adquisición de los dispositivos GPRS, RFID y de los componentes electrónicos que permitan la construcción del hardware que hará posible la correcta interacción de los subsistemas que componen el sistema.

- Desarrollo del hardware y del software, necesarios para la materialización de la propuesta.
Pruebas del sistema y ajustes finales, que determinen la pertinencia del uso de este sistema en otros servicios que se prestan a los estudiantes.

A continuación se describe el diseño del sistema mencionado.

\section{DISEÑO DEL PROTOTIPO}

Con base en los requerimientos planteados y en el sistema conceptualizado en la Figura 1, se procede a la selección de cada una de las partes que componen el sistema. Se añade a las partes anteriormente mencionadas, una impresora, que dará al estudiante un comprobante impreso del servicio prestado, y que servirá como documento de soporte en caso de que haya alguna reclamación o se presente un evento no contemplado en los diseños preliminares. De esta forma, usando como criterios de selección de los equipos y del hardware: la disponibilidad y representación en el mercado nacional, la relación costo beneficio de los equipos, la documentación y el soporte disponible y los recursos con que cuenta el grupo GIRA-UPTC, se adquieren los dispositivos requeridos que se mencionarán a continuación.

\subsection{Descripción de los equipos empleados}

Como sistema de lectura escritura RFID, con base en los criterios previamente mencionados, se seleccionó el dispositivo PT-850, importado a Colombia por la empresa PLINTEC (pág. web Plintec.com). Este lector posee una antena interna de alto rendimiento, que proporciona una solución simple y de bajo costo. Adicionalmente, el dispositivo permite la lectura de múltiples tag de forma simultánea, siempre que estén ubicados en la zona de cobertura del lector. El PT-850 cuenta con protocolos de intercambio de datos Ethernet, comunicación RS-232 y entradas y salidas con niveles TTL, especialmente adaptadas para el uso de microcontroladores (Alcalá, 2003).

Las características generales del lector de RFID, son:

- Frecuencia de operación de 13.56 Mhz.

- Fuente conmutada de voltaje $(5 \mathrm{~V}, 1.2 \mathrm{~A})$.

- Cable de comunicación serial.

- Software de entrenamiento.

- Transponder Vicinity Card (tipo tarjeta)

El lector RFID seleccionado, aparece en la Figura 2.

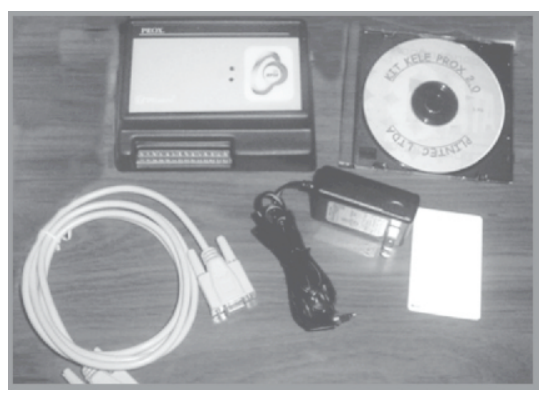

Figura 2. Lector RFID PT-850. 
Para la impresión de los recibos destinados a los estudiantes, se seleccionó la impresora portátil de referencia MO9530RX de la empresa Starmicronics (pág. web starmicronics.com), ya que este modelo permite fácil comunicación con un microcontrolador e impresión sin tinta, pues emplea calor y elimina la necesidad de cambio de cartuchos. Debe señalarse que las impresoras portátiles dan la posibilidad de contar con los recursos de sus contrapartes de escritorio, pero con la ventaja de la facilidad en el transporte, dado su reducido volumen y masa, lo que permite trasladarlas de forma simple. Dentro de las características de la impresora seleccionada, están:

Velocidad de 2 IPS.

Fuente $9 \mathrm{~V}$.

Puertos serial, infrarrojo, bluetooth.

Funcionamiento con batería recargable de 10 A.h.

Alimentación automática de papel.

En la Figura 3 se aprecia la impresora seleccionada, de acuerdo con los criterios señalados de forma previa.

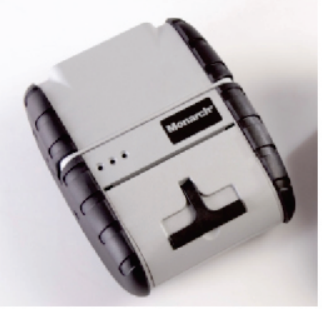

Figura 3. Impresora MO9530RX.

Para la comunicación con el servidor web, se seleccionó el módem GPRS Q64, de bajo costo, fácil manejo y puertos de comunicación compatibles con estándares manejados por diferentes familias de microcontroladores. Este módulo permite enviar y recibir datos de un equipo que pueda comunicar comandos AT mediante un puerto serial estándar (Jain, 2010). De esta forma se pueden usar las redes celulares para el transporte de datos. Las características del dispositivo seleccionado son:

- Dieciséis salidas digitales para intercambio de datos en formato paralelo síncrono.

- Puertos serial RS 232, USB e I2C, para comunicación con microcontroladores.

- Operación con 3.3V.

- SMS, CSD, GPRS clase 10.

En la Figura 4 se puede apreciar una imagen del módem GPRS Q64.

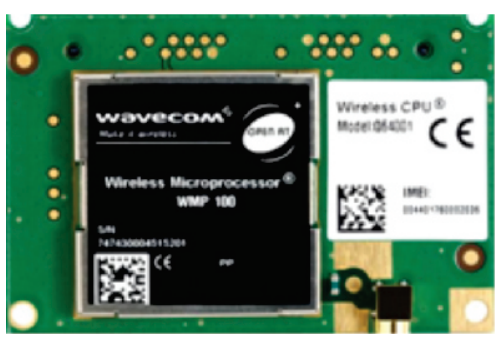

Figura 4. Módem GPRS Q64

\subsection{Servidor web implementado}

Por otra parte, el servidor implementado se encarga de recibir, almacenar y llevar a cabo todos los procesos de consulta de la información del sistema, cuando atiende a alguno de los estudiantes registrados. El servidor está conectado a Internet y tiene una dirección IP pública, de esta forma el módulo de consulta móvil tiene registrada esta dirección y podrá comunicarse con él. Adicionalmente, el servidor web tiene instalada una aplicación que recibe las peticiones de la estación base, que atiende las solicitudes realizadas por los estudiantes identificados mediante el tag RFID. Hay que agregar que la aplicación mencionada emplea un socket y la pila TCP/IP, para garantizar la integridad de la información, con acceso a una base de datos para el depósito de la misma, procedente de la red. Además se cuenta con una aplicación auxiliar que permite desplegar los datos acumulados, para ser impresos o visualizados por parte del administrador del sistema.

Hay que tener en cuenta que dados los limitados recursos económicos disponibles en el grupo GIRA-UPTC, no fue posible alojar la aplicación web en un servidor comercial, por esta razón se utilizó un computador con acceso a Internet como servidor web, en el que se instalaron las aplicaciones elaboradas.

Para la elaboración de las aplicaciones del servidor web, se usó Visual Basic 6.0, ya que este paquete permite de forma sencilla y rápida la creación de software para dar servicios de información a través de Internet (Internet Information Services IIS). Esta característica de Visual Basic es una de las posibilidades que este ambiente de desarrollo presenta al programador, como se aprecia en la Figura 5.

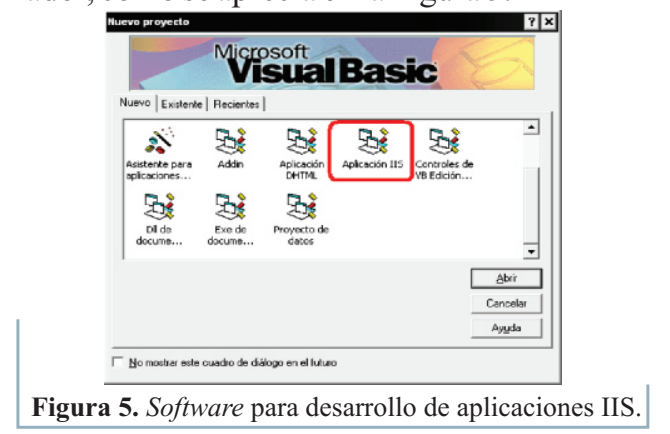

Para ejecutar aplicaciones IIS, se instaló el servicio Personal Web Server en el sistema sobre el cual se ejecutaron las aplicaciones elaboradas, y se usó como sistema operativo el Windows XP Profesional. Adicionalmente, en el servidor se alojaron las siguientes aplicaciones que hicieron posible implementar el sistema: el motor de la base de datos, la aplicación de comunicaciones y la aplicación web.

El motor de la base de datos se usa para almacenar la información correspondiente a cada estudiante. El administrador del sistema está en capacidad de actualizar dicha información o agregar nuevos registros. Previendo la cantidad de información por guardar y la frecuencia y modo de acceso a ella, se decidió utilizar SQL Server (Structured Query Language) que se ejecuta sobre un servidor local (Labordere, 2004). 
La aplicación de comunicaciones administra las sesiones de transmisión del módulo móvil de consultas, para lo que deben preverse conexiones simultáneas. Básicamente, el proceso consiste en abrir un puerto y esperar solicitudes de conexión. Al establecerse una conexión (en el socket), se utiliza un mecanismo de reconocimiento del cliente que puede estar basado en métodos tales como claves de acceso simétricas, para determinar el permiso de mantener la conexión, autenticando el módulo móvil y luego recibiendo la información entrante (código ingresado). Posteriormente se envía la información saliente (saldo) y finalmente termina la conexión liberando recursos para atender otra consulta. Al terminar una comunicación iniciada por una solicitud de consulta, se incluye en esta aplicación la capacidad de actualizar el registro "saldo" en la base de datos, para poner al día la información. Para este caso se desarrollaron módulos WebClass en la aplicación IIS (Wakerly, 2001).

La aplicación web da la posibilidad a los usuarios de hacer consultas a través de Internet con un sitio en la red dispuesto para tal fin. La página inicial indica al usuario que debe identificarse para tener acceso a la consulta del saldo, que es de uso libre por parte de los estudiantes. Si se tienen privilegios de administrador, se puede acceder a los registros de los estudiantes y aplicar cambios a los mismos. En la Figura 6 se aprecia la pantalla inicial de la aplicación web desarrollada.

\subsection{Hardware y software desarrollado}

El módulo estudiante indicado en la Figura 1, consta del lector RFID, que permite al estudiante identificarse para usar el sistema cuando se accede al saldo (uso que genera un costo). El sistema se conecta con la base de datos, consulta el saldo y descuenta del registro del usuario en particular, además se puede visualizar el nuevo saldo a través de una pantalla de cristal líquido alfanumérico (Tocci, 2008).

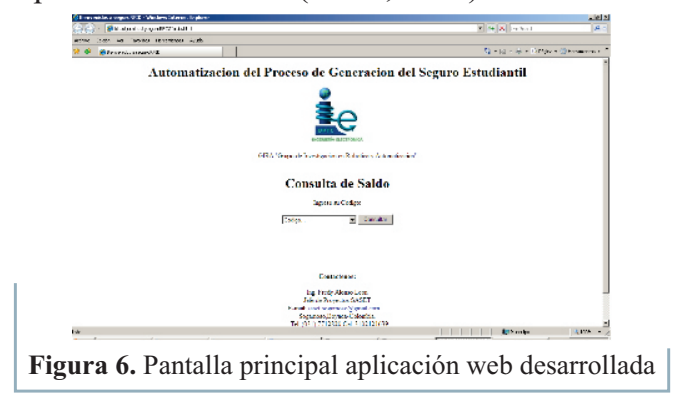

El corazón de este módulo es el microcontrolador PIC16f877A de la empresa Microchip. Este hace uso del módem Q64 para comunicarse con la base de datos y dar servicio al usuario que recién se ha identificado a través del enlace RFID. Adicionalmente, el estudiante cuenta con la posibilidad de acceder a la base de datos mediante un teclado matricial y el enlace GPRS, para consultar el saldo sin necesidad de identificarse con el tag RFID, pero sí debe digitar de forma previa un código de seguridad, único para cada usuario. El diagrama de bloques del módulo estudiante aparece en la Figura 7.

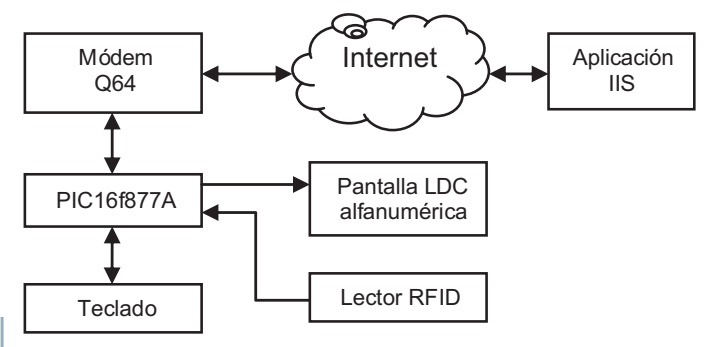

Figura 7. Diagrama de bloques de módulo que presta atención a los estudiantes.

Como se puede apreciar en la Figura 7, el enlace entre el usuario y la aplicación web, se efectúa a través del módem GPRS Q64 de la empresa Wavecom, conectado a un microcontrolador Microchip PIC16F877A (Angulo, 2000), que cuenta con un firmware desarrollado en código ensamblador, mediante la aplicación de uso libre MPLAB IDE versión 8.0 de la empresa Microchip (pág. web Microchip.com) (Morris, 2003). El hardware desarrollado para el módulo estudiante aparece en la Figura 8 .

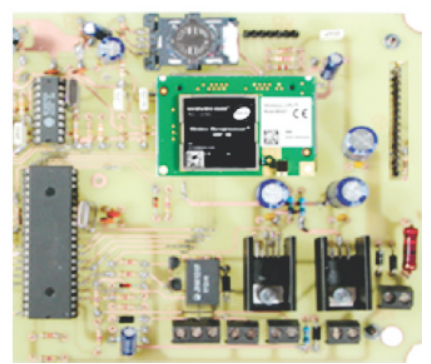

Figura 8. Imagen del hardware para integrar los periféricos del módulo desarrollado para atender a los estudiantes.

El periférico del microcontrolador, compuesto por la pantalla LCD alfanumérica y el teclado matricial, permite hacer consultas a los estudiantes de forma flexible, sin contar con un tag RFID, pero con el requerimiento de un código de validación (Mitzner, 2007). Este periférico aparece en la Figura 9.

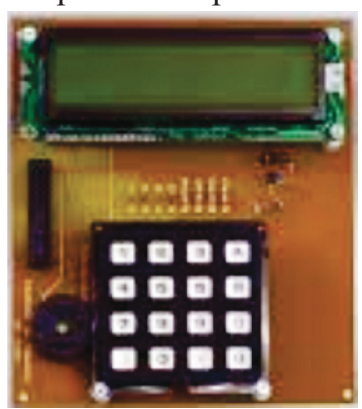

Figura 9. Periférico para permitir consultas sin uso de tag RFID.

Para inicializar el módem Q64, se usan rutinas de inicialización alojadas en la memoria de programa del microcontrolador. Estas rutinas, adicionalmente controlan el sistema de suministro de energía del módem y su restablecimiento.

La rutina de inicialización del módem se describe así: el microcontrolador envía un comando AT al módem para desactivar la retransmisión byte por byte de la UART (Universal Asynchronous Receiver Transmitter) (Cutler, 1990). 
Adicionalmente se detecta el estado activo del módem. Si no se recibe respuesta al comando, después de haber esperado durante un tiempo determinado (timeout) se reenvía el comando una vez más. Si después del segundo intento nuevamente expira el timeout, se asume que el módem no está conectado al microcontrolador y se ejecuta una rutina de indicación de no conexión al usuario (Lautenschlager, 2000). La rutina de inicialización del módem es representada por el diagrama de flujo de la Figura 10.

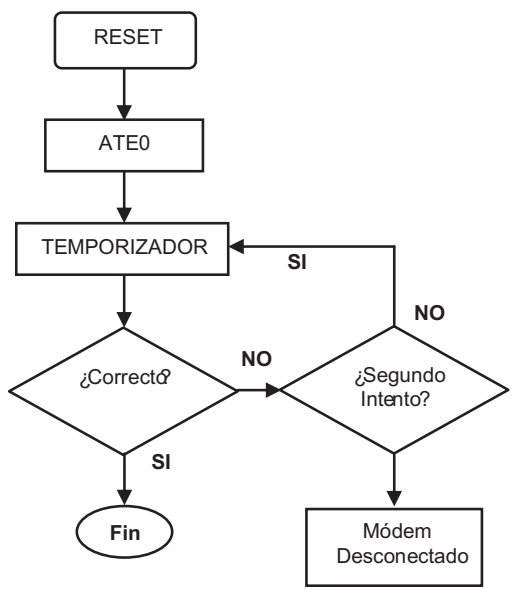

Figura 10. Diagrama de flujo de la rutina de inicialización del módem Q64

la red no acepta el módem, o que este ya está registrado con una dirección IP. Para el funcionamiento del Q64, se usa una dirección IP alojada en la memoria EEPROM del microcontrolador (Mandado, 2007). El diagrama de flujo de las rutinas de registro del módem Q64 implementadas, se muestra en la Figura 11.

La rutina se repite con periodos de latencia preestablecidos, que toman como referencia el momento en que el microcontrolador se reinicia o el instante en que se hizo la última transmisión. Los intervalos son programados en el firmware del microcontrolador y pueden ser modificados por software.

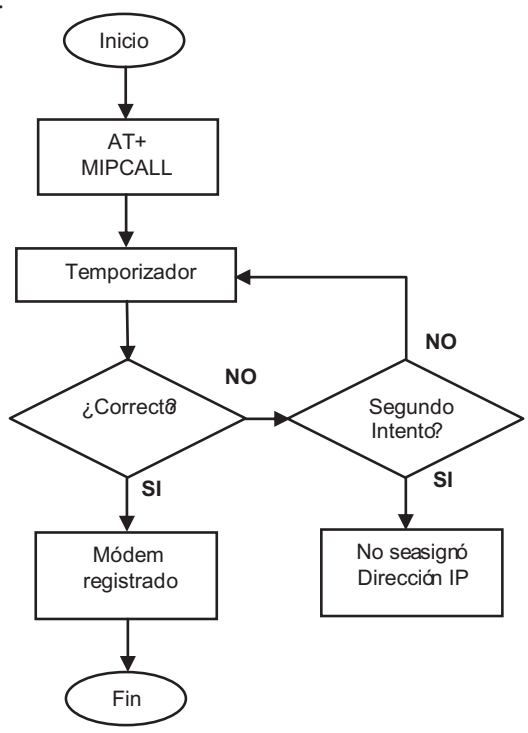

Figura 11. Diagrama de flujo de la rutina de registro del módem Q64.
A continuación se describe el proceso de registro, apertura de socket y envío de datos: el microcontrolador envía comandos al módem para hacer la petición de abrir un socket en el servidor, si no se recibe respuesta al comando, después de haber esperado durante un tiempo determinado (timeout) (Tomasi, 2003), se incrementa el periodo de latencia durante un nuevo timeout y se vuelve a enviar la petición de apertura de socket. Este bucle se repite tres veces; en caso de que no se consiga la apertura, se reinicia el módem, de esta manera se reduce la posibilidad de falla por enclavamiento lógico del Q64 (Cutcher, 2005).

Si se consigue la apertura del socket, se pasa a la rutina de autenticación; en esta, el móvil envía un código que incluye su número de identificación. Si el servidor reconoce el código, mantiene la conexión y devuelve una confirmación; en caso contrario, se infiere que se trata de una conexión no deseada y directamente cierra el socket para evitar intrusiones (Quintana, 2008).

Una vez identificado, el módem Q64 comienza la secuencia de transmisión que consta de 26 paquetes, donde se incluye la información de las entradas y salidas del microcontrolador, texto de intercambio y fecha, entre otros. Por último, se cierra el socket y se ponen en cero los contadores para determinar el tiempo en que será necesaria la transmisión siguiente (Tisal, 2005). El diagrama de flujo de este proceso aparece descrito en la Figura 12.

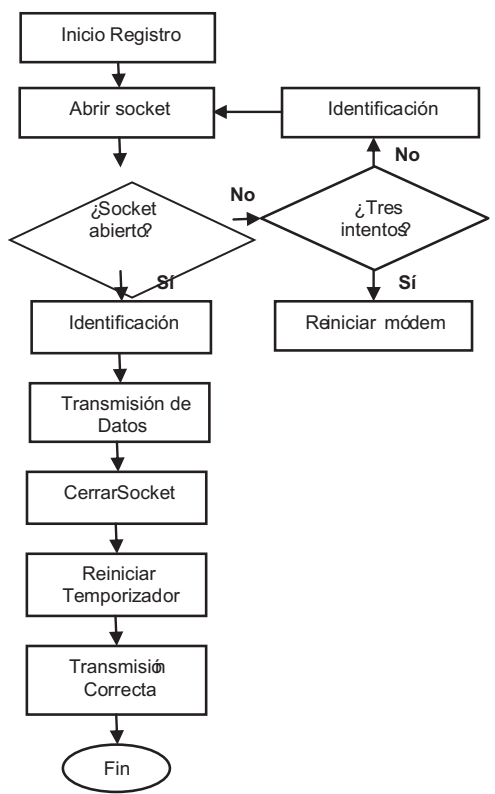

Figura 12. Diagrama de flujo de la rutina de apertura de socket y envío de datos a servidos web mediante el módem Q64.

\section{RESULTADOS DE LA PRUEBA DE DESEMPEÑO DEL PROTOTIPO}

Las pruebas del prototipo fueron exitosas, pues este funcionó según lo esperado y se demostró que la tecnología RFID puede reemplazar los sistemas de códigos de barras, pero con un nivel mucho mayor de seguridad. 
En efecto, la lectura de los tag brindó una fiabilidad de cerca del $100 \%$, con la ventaja observada que estos siguieron operando, aun cuando sus usuarios no tuvieron ningún cuidado en el manejo de los dispositivos de identificación. La prueba piloto fue realizada con lectores ubicados en la oficina del grupo de investigación GIRA, y los diferentes tag fueron distribuidos entre algunos de los estudiantes del semillero de investigación. La prueba del prototipo se llevó a cabo a lo largo del mes de marzo del año 2010.

Una ventaja adicional de esta tecnología, es que no hay necesidad de línea de vista para la lectura de los tag, lo que permite inferir que el usuario no requiere sacar de su bolsillo su identificación, pues, sencillamente con acercarse al lector RFID, es registrado por el sistema. Además, a diferencia de los lectores que usan código de barras, en condiciones medioambientales y de mantenimiento adversas, la tecnología RFID logró una tasa de lecturas exitosas del $99.5 \%$, contra un $90 \%$ de lecturas exitosas logradas con un sistema de lectura similar, pero que usó identificación por medios ópticos, sin la necesidad de que el tag fuera visible en la superficie del lector.

Además, los componentes RFID operaron en variadas condiciones ambientales, incluyendo temperaturas de menos de cinco grados centígrados y temperaturas de más de 60 grados centígrados, expuestos a la radiación directa del sol en días muy calurosos. Asimismo, los tag fueron sometidos a distintas condiciones adversas de humedad, lluvia, vibraciones, hielo y contaminación por grasa y otros agentes presentes en el ambiente en que interactúan los estudiantes, obteniendo tasas de lectura satisfactoria por encima del $99 \%$. Para analizar el funcionamiento de los tag en condiciones en que son expuestos a diferentes fuentes de radiación, como señales de radio de teléfonos celulares, se ubicó el lector sobre la antena del módem Q64, y la lectura de los dispositivos RFID no se vio afectada.

Por otra parte, se menciona que el sistema de consultas que usó la red GSM mediante el módem Q64 y el servidor web conectado a Internet, resultó ser una valiosa herramienta, de bajo costo y elevadas prestaciones, pues se lograron consultas en la base de datos desde los lugares de la red que se pusieron a prueba y se pudieron instalar sistemas de lectura RFID en cualquier lugar en que el operador de telefonía celular tuvo cobertura, que, en nuestro contexto, puede ser toda la geografía nacional e incluso fuera de ella. El sistema de comunicación implementado mediante GPRS fue aplicado con éxito en otros sistemas de monitoreo y control que no involucraron necesariamente Internet.

\section{CONCLUSIONES}

Se desarrolló un sistema compuesto por hardware y software, elaborado por el grupo de investigación GIRA de la UPTC, que se convierte en una alternativa viable para la automatización de los procesos administrativos tanto en el interior de la universidad como fuera de ella.
El estándar GPRS ofrece un enlace inalámbrico seguro para la transmisión de información, gracias a la encriptación y autenticación inherente al protocolo, y además es una buena alternativa para acceder sistemas de telemonitoreo a través de Internet, desde cualquier lugar donde haya cobertura de las redes celulares.

El uso de las bases de datos alojadas en un servidor web, sumado a la comunicación en tiempo real con los lectores RFID mediante el módem Q64, permite que el sistema mantenga actualizada toda la información, y le brinde al administrador y a los usuarios, la posibilidad de efectuar procesos de consulta, apertura, actualización y eliminación de registros de usuarios y generación de reportes actualizados; en otras palabras, hace posible un uso eficaz y eficiente del sistema construido.

\section{RECOMENDACIONES Y TRABAJOS FUTUROS}

Se recomienda el uso del hardware y el software elaborados, en aplicaciones que involucren la agricultura de precisión y los procesos de monitoreo y seguridad en minería, pues en la actualidad están haciendo falta en los sistemas de producción del departamento de Boyacá.

El sistema gestado puede ser integrado a sistemas de acceso de personal a diferentes instituciones, por lo que puede ser utilizado para incrementar el nivel de seguridad de los bienes de la universidad, controlando el ingreso de visitantes a la institución.

Se aconseja profundizar sobre la integración de sistemas GPRS a servidores web, ya que su combinación se puede emplear en infinidad de sistemas que agilicen procesos administrativos, tanto en el interior de la universidad como fuera de ella.

Se sugiere usar el sistema elaborado, como base para futuras investigaciones que exploten la potencialidad de las redes celulares en el transporte de información.

Los sistemas RFID pueden ser aplicados en situaciones en que se requiera identificación en condiciones hostiles, sin ningún tipo de línea de vista, lo que hace de esta tecnología, una alternativa viable en aplicaciones de robótica, especialmente en los proyectos de robótica educativa, ya que con los tag, los niños consiguen asociar diferentes conceptos y dominios simbólicos que pueden ser representados de variadas formas.

\section{AGRADECIMIENTOS}

El Grupo de Investigación en Robótica y Automatización Industrial GIRA-UPTC, agradece a las directivas de la Universidad y a la DIN, por el apoyo en la ejecución del presente trabajo; a los estudiantes del semillero de investigación por la colaboración en la validación del prototipo implementado, y a la Escuela de Ingeniería Electrónica, por respaldar la propuesta en todas sus fases. 


\section{REFERENCIAS}

Angulo, U. (2000). Microcontroladores PIC: diseño práctico de aplicaciones. ( $2^{\mathrm{a}} \mathrm{ed}$.). España: McGraw Hill.

Alcalá, J. J. \& Maxines, D. G. (2003). VHDL El arte de programar sistemas digitales. México: Patria.

Cutcher, D. (2005). Electronic circuits for the genius. EE. UU.: Tab Electronics.

Cutler, P. (1990) Análisis de circuitos con semiconductores. ( $2^{\mathrm{a}}$ ed.). México: McGraw Hill.

Floyd, T. L. (2008). Fundamentos de sistemas digitales. ( $9^{\mathrm{a}}$ ed.). España: Pearson Educación.

Garza, G. J.A. (2006). Sistemas digitales y electrónica digital, prácticas de laboratorio. España: Pearson Educación.

Jain, R. P. (2010). Modern digital electronics. ( $4^{\text {th }}$ ed.). New Delhi: Mc Graw Hill.

Labordere, A. (2004). SMS and MMS interworking in mobile networks. $\left(2^{\text {nd }}\right.$ ed.). Inglaterra: Artech House Publisher.
Lautenschlager, G. (2000). Móviles SMS WAPy Compañia. $\left(1^{\text {st }}\right.$ ed.). España: McGraw Hill.

Mandado, P. E. (2007). Sistemas electrónicos digitales. $\left(9^{\text {th }}\right.$ ed.). España: Marcombo.

Mitzner, K. (2007). Complete PCB design using OrCad capture and layout. EE.UU.: Elsevier.

Morris, M. (2003). Diseño digital. México: Pearson Educación.

Quintana, G. (2008). Aprende SQL. España: Publicaciones Universitat Jaume.

Tisal, J. (2005). La red GSM. (1 ${ }^{\text {a }}$ ed.). España: Paraninfo.

Tocci, R. J., Widmer, N. S. \& Moss, G. L. (2007). Sistemas digitales, principios y aplicaciones. $\left(10^{\mathrm{a}} \mathrm{ed}.\right)$. México: Pearson Educación.

Tomasi, W. (2003) Sistema de comunicaciones electrónicas. (4 $4^{\mathrm{a}}$ ed.). México: Prentice Hall.

Wakerly, J. F. (2001). Diseño digital: principios y prácticas. México: Prentice Hall. 